\title{
Can Meditation Influence Quality of Life, Depression, and Disease Outcome in Multiple Sclerosis? Findings from a Large International Web-Based Study
}

\author{
Adam B. Levin, ${ }^{1}$ Emily J. Hadgkiss, ${ }^{2}$ Tracey J. Weiland, ${ }^{1,2}$ Claudia H. Marck, ${ }^{2}$ \\ Dania M. van der Meer, ${ }^{2}$ Naresh G. Pereira, ${ }^{3}$ and George A. Jelinek ${ }^{2,4}$ \\ ${ }^{1}$ School of Medicine, The University of Melbourne, Melbourne, VIC 3010, Australia \\ ${ }^{2}$ Emergency Practice Innovation Centre, St. Vincent's Hospital, Melbourne, VIC 3065, Australia \\ ${ }^{3}$ Department of Epidemiology and Preventive Medicine, Monash University, Prahran, VIC 3800, Australia \\ ${ }^{4}$ Medical School, University of Notre Dame, Fremantle, WA 6959, Australia \\ Correspondence should be addressed to Adam B. Levin; a.levin@student.unimelb.edu.au
}

Received 11 May 2014; Revised 9 September 2014; Accepted 14 October 2014; Published 12 November 2014

Academic Editor: Frederic Blanc

Copyright (C) 2014 Adam B. Levin et al. This is an open access article distributed under the Creative Commons Attribution License, which permits unrestricted use, distribution, and reproduction in any medium, provided the original work is properly cited.

\begin{abstract}
Objectives. To explore the association between meditation and health related quality of life (HRQOL), depression, fatigue, disability level, relapse rates, and disease activity in a large international sample of people with multiple sclerosis (MS). Methods. Participants were invited to take part in an online survey and answer questions relating to HRQOL, depression, fatigue, disability, relapse rates, and their involvement in meditation practices. Results. Statistically and potentially clinically significant differences between those who meditated once a week or more and participants who never meditated were present for mean mental health composite (MHC) scores, cognitive function scale, and health perception scale. The MHC results remained statistically significant on multivariate regression modelling when covariates were accounted for. Physical health composite (PHC) scores were higher in those that meditated; however, the differences were probably not clinically significant. Among those who meditated, fewer screened positive for depression, but there was no relationship with fatigue or relapse rate. Those with worsened disability levels were more likely to meditate. Discussion. The study reveals a significant association between meditation, lower risk of depression, and improved HRQOL in people with MS.
\end{abstract}

\section{Introduction}

Multiple sclerosis (MS) is a common chronic neurological disease $[1,2]$ typified by neuronal demyelination and inflammation, leading to axonal injury with disease progression [2]. There are several categories of MS, with relapsingremitting being the most common [3]. MS is currently incurable; however, there are many strategies used for disease management, including pharmacological agents, lifestyle modification, rehabilitation, psychosocial support, and rarely surgery, all of which have varying efficacy [4-6].

It has been proposed that stress may play a role in the MS disease course, given that it has been shown to have a strong association with frequency of disease relapses $[7,8]$. It is thought, therefore, that strategies that reduce and manage stress may play a role in secondary or tertiary prevention for people with MS by slowing disease course and improving quality of life (QOL). One such strategy is meditation. Interest in meditation as a medical treatment was generated by Kabat-Zinn's research showing that meditation could be a useful tool for managing chronic pain [9]. Meditation, which encompasses a wide range of techniques, has been associated with symptom reduction in psychiatric conditions, electroencephalography (EEG) changes and beneficial structural brain changes on neuroimaging with long term use [10-18]. More specifically meditation has been associated with stress reduction, decreased rate of maladaptive coping strategies, and increased resilience for those with MS [19]. 
A randomised controlled trial by Grossman et al. is, to date, the strongest evidence for the efficacy of meditation on MS morbidity outcomes [20]. In the study 150 participants were randomised into a mindfulness based intervention program or a control group of standard treatment, with those in the experimental group having significantly improved health related quality of life (HRQOL) as well as decreased depression, anxiety, and fatigue up to six months after the intervention. Other interventional studies have also found clinically significant benefits of meditation on MS [21-24]; however these studies were not as methodologically rigorous as that led by Grossman.

Further evidence to support the use of meditation as a successful management adjunct for MS exists primarily in the form of cross-sectional studies. These studies also highlight a positive association between meditation and HRQOL in MS; however they are often limited by difficulty in verification of the MS diagnosis [25-27], being limited to one or two countries $[19,25,27,28]$, not employing validated instruments [26-28], and small sample size [19, 28].

While some evidence exists that meditation may have a beneficial effect on HRQOL for those with MS, none of the current literature has demonstrated any significant positive effect on decreasing relapse rates. This study is part of a research project "Health Outcomes and Lifestyle Interventions in a Sample of People with Multiple Sclerosis" (the HOLISM study). Previous studies published from the same dataset have examined the association between health related outcomes in MS, omega 3 supplementation and fish consumption [29], other dietary factors [30], and alcohol and smoking [31]. The aim of this study was to explore the relationship between meditation frequency and HRQOL, depression, fatigue, disability status, relapse rate, and disease activity in a large international sample of people with MS.

\section{Methods}

2.1. Participants and Recruitment. Participants were asked to complete an online survey on SurveyMonkey, reading a participation statement and giving consent prior to starting the survey. Participants aged 18 years or over who were diagnosed with MS by a physician were invited to take part. Ethics approval was granted by St. Vincent's Hospital Melbourne Human Research Ethics Committee (LRR 055/12). More detailed methodology has previously been described [32].

2.2. Data Collection and Tools. The study collected demographic details, including age, gender, education status, and current employment status, and diagnostic details, including type of MS, whether MS was diagnosed by a clinician, and years since diagnosis. All data were self-reported.

2.3. Health Related Quality of Life. The Multiple Sclerosis Quality of Life-54 survey (MSQOL-54) was used to measure HRQOL. This well validated tool comprises the 36-item short form health survey (SF-36) and 18 disease specific questions. 54 items give rise to 12 scales, 2 separate items, and a mental health composite (MHC) and a physical health composite (PHC) [29, 33-35]. Physical and mental health composites and subscales were calculated as per the scoring method from the MSQOL-54.

2.4. Disability. Disability was assessed using the PatientDetermined Disease Steps (PDDS), a tool that has been used by the North American Research Committee on Multiple Sclerosis registry (NARCOMS) as a surrogate for the Expanded Disability Status Scale (EDSS) [36-39]. The EDSS is commonly used to assess gait disability and correlates well with the PDDS which scores level of disability from 0 (normal) to 8 (bed bound). The PDDS was collapsed to form three ordinal categories: mild (scores 0-2), moderate (3-5), and major disability (6-8).

2.5. Relapse Rate. Participants were asked to recall how many medical doctor-diagnosed MS relapses they had in the previous 12 months and five years. A categorical variable "disease activity" was created by assessing whether 12-month relapse rate was higher, the same, or lower than participants' 5 -year annualised relapse rates. For the purpose of analysis, only people with a relapsing-remitting type of MS were included for comparison of relapse rate or disease activity.

2.6. Depression. Depression risk was assessed using the twoitem Patient Health Questionnaire-2 (PHQ-2), a shortened version of the PHQ-9, which has been validated in an MS population. This tool has a reported specificity of $92 \%$ and sensitivity of $83 \%$ for major depression with a cut-off score $\geq$ 3 [40].

2.7. Fatigue. Fatigue was assessed using the Fatigue Severity Scale (FSS), a tool which has been validated for use in an MS population [41, 42]. The FSS is a nine-question survey with answers being scored from 0 (least severe) to 7 (most severe). A total score above 4 was considered an indication of clinically significant fatigue, as determined in the literature [43].

2.8. Meditation. Participants were asked to provide an average weekly frequency of meditation in the last 12 months with responses including never, less than once per week, once to twice a week, three to four times a week, five to six times a week, and every day.

Meditation frequency variables were collapsed for meaningful analysis:

(1) collapsed to two categories-never meditates or has meditated in the last 12 months;

(2) collapsed to three categories-never meditates, meditates less than once per week, or meditates one or more times per week.

No specific definition of meditation was provided to participants who were simply asked how often and for how long they participated in meditation. 
2.9. Data Analysis. Data were analysed using IBM SPSS version 21.0.

Only participants reporting that their diagnosis had been confirmed by a medical doctor were included in the analysis; those with clinically isolated syndrome or possible MS were excluded.

Categorical demographic data were analysed using cross tabulation to create contingency tables with Pearson's Chi Square or Fisher's Exact Test and adjusted standardised residuals (ASR) used to determine over- and underrepresentation of specific demographic groups participating in meditation. Results were only accepted if all cells had an expected cell count greater than 5 and results were considered significant if ASR were $\geq 2$ or $\leq-2$ and alpha was $<0.05$.

Bivariate analyses were undertaken to explore the relationship between meditation and each variable. When analysing 12-month relapse rates only those who had relapsing-remitting MS and had relapses confirmed by a physician were included.

T-tests were used to analyse significant differences in continuous variables between those that did and did not meditate (dichotomous variable), using Levene's test for assessment of equal variance.

Analysis of the categorical meditation data was undertaken using cross tabulation with categorical data (as described above) and one way ANOVA for comparison with continuous data. For all ANOVA, homogeneity of variance was assessed with Levene's test. Post hoc analyses were performed for significant group differences using Tukey HSD or Games Howell to assess paired differences, depending on homogeneity of variance.

Multivariate analysis was performed to determine independent predictor variables for outcome variables that were significant in bivariate analyses: MSQOL-54 composites, PDDS, and PHQ-2. Multiple regression (Enter method) was used for MSQOL-54 composites. Tests were performed to assess normality, linearity, and homoscedasticity and to check for outliers. Absence of multicollinearity was assessed with variance inflation factor $(<4)$ and correlations $(<0.7)$. Binary logistic regression was used for PHQ-2 with goodness of fit being assessed with omnibus and Hosmer and Lemeshow test. Ordinal logistic regression was used to assess PDDS. Assumptions were tested using: proportional odds assumption (test of parallel lines > 0.05), goodness of fit, and multicollinearity as above. Significance was assumed if twotailed tests were below 0.05 .

Given the large sample size there were enough participants to satisfy the requirements for all the tests used. Similarly, the central limit theorem was assumed for analyses involving continuous data.

\section{Results}

3.1. Demographics. There were 3132 respondents to the HOLISM study overall, and 2469 fit the inclusion criteria of being 18 years or over and having physician diagnosed MS. Of these $82.3 \%$ were female. The median age was 46 years $(\mathrm{IQR}=30.5-60.5)$ and median age at diagnosis 37

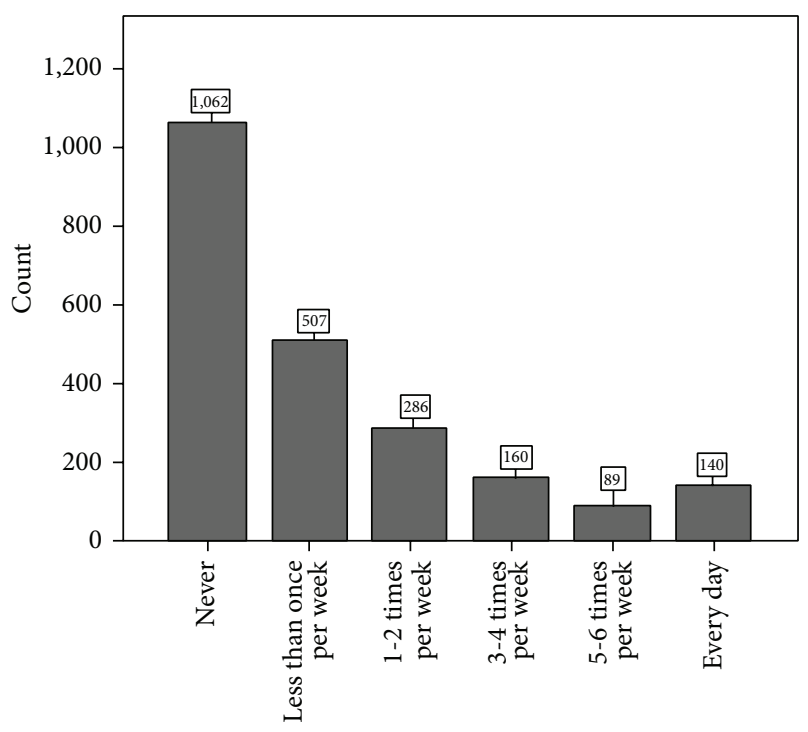

On average in the last 12 months, how often have you meditated?

FIGURE 1: Meditation frequency of survey respondents.

years (IQR $=22-52$ ). Almost two-thirds of respondents had relapsing-remitting MS. Respondents were largely from the USA (32.7\%), Australia (25.5\%), and the UK (16.9\%). Further demographic data from the HOLISM have been published previously [32].

Of the 2469 respondents, up to 2244 (90.9\%) completed the meditation section of the HOLISM survey. 1182 (52.7\%) reported having meditated at least once in the past twelve months (Figure 1).

Women, those with bachelor degrees or higher, those working part time, and those in the age category $50-59$ years were all more likely to have participated in meditation in the past twelve months (Table 1). There was no statistically significant association between meditation status and years since diagnosis of MS (data not shown).

3.2. HRQOL. PHC and MHC scores were significantly higher in respondents who meditated compared to those who did $\operatorname{not}(P=0.001$ and $<0.001$, resp.). Similarly the overall quality of life, energy, health distress, emotional well-being, health perception, and cognitive function HRQOL scales were all significantly higher in those who meditated $(P<0.001)$. Sexual health, pain, and social function HRQOL subscore mean differences were not related to meditation (data not shown).

Analyses showed a significant difference in MHC and PHC for frequency of meditation. Post hoc comparisons (Table 2) showed participants who meditated once or more per week had a higher MHC mean score compared to those who never meditated and those who meditated less than once per week, both of which were statistically significant. There was no significant difference between those who meditated less than once a week and those who never meditated. Identical analysis of the PHC demonstrated a significantly higher mean PHC score for participants who meditated once 
TABLE 1: Summary of demographics comparing those who meditated in the last 12 months to those who did not.

\begin{tabular}{|c|c|c|c|c|}
\hline & & & & \\
\hline & Yes, $n(\%)$ & No, $n(\%)$ & Total (\%) & $P$ \\
\hline Gender & & & & \\
\hline Female & $981(53.5)^{*}$ & $852(46.5)^{\dagger}$ & $1833(100)$ & 00404 \\
\hline Male & $176(45.5)^{\dagger}$ & $211(54.5)^{*}$ & $387(100)$ & 0.004 \\
\hline Age & & & & \\
\hline $18-29$ years old & $50(44.2)$ & $63(55.8)$ & $113(100)$ & \\
\hline $30-39$ years old & $291(52.1)$ & $268(47.9)$ & $559(100)$ & \\
\hline $40-49$ years old & $348(49.1)$ & $361(50.9)$ & 709 (100) & 0.042 \\
\hline $50-59$ years old & $322(55.7)^{*}$ & $256(44.3)^{\dagger}$ & $578(100)$ & \\
\hline $60+$ years old & $127(56.2)$ & $99(43.8)$ & $226(100)$ & \\
\hline Education & & & & \\
\hline Did not complete school & $17(37)^{\dagger}$ & $29(63)^{*}$ & $46(100)$ & \\
\hline Completed school/trade & $376(44.3)^{\dagger}$ & $472(55.7)^{*}$ & $848(100)$ & $<0.001$ \\
\hline Bachelor degree or higher & $775(57.8)^{*}$ & $566(42.2)^{\dagger}$ & $1341(100)$ & \\
\hline Employment & & & & \\
\hline Work full time & $353(48.2)^{\dagger}$ & $380(51.8)^{*}$ & $733(100)$ & \\
\hline Work part time & $285(59.5)^{*}$ & $194(40.5)^{\dagger}$ & $479(100)$ & \\
\hline Stay-at-home parent/carer & $92(53.8)$ & $79(46.2)$ & $171(100)$ & \\
\hline Unemployed & $88(52.4)$ & $80(47.6)$ & $168(100)$ & 0.013 \\
\hline Retired due to disability & $267(51.9)$ & $247(48.1)$ & $514(100)$ & \\
\hline Retired due to age & $37(52.1)$ & $34(47.9)$ & $71(100)$ & \\
\hline Other (incl. students) & $49(47.6)$ & $54(52.4)$ & $103(100)$ & \\
\hline
\end{tabular}

* denotes significantly overrepresented as determined by standardised adjusted residuals.

${ }^{\dagger}$ denotes significantly underrepresented as determined by standardised adjusted residuals.

or more per week compared to those who never meditated and for those who meditated less than once per week, when compared to those who never meditated. There was no significant difference between those who meditated less than once a week and those who meditated at least once per week (Table 2).

Multiple regression analysis was conducted to predict PHC and MHC from age, gender, education level, and frequency of meditation. The models generated accounted for $8.1 \%$ of PHC variance and 3.0\% of MHC variance (Table 3).

After adjusting for age, gender, and education, participants who meditated once or more per week had a significantly higher mean MHC compared to those who never meditated, whereas there was no significant difference between participants who meditated less than once a week and those that never meditated $(P<0.001$ and $P=0.513$, resp.). The same adjustments for $\mathrm{PHC}$ found that the mean score was significantly higher for those who meditated once or more per week or less than once per week compared to those who never meditated ( $P=0.009$ and $P=0.031$, resp.). However, there was no significant difference between the two categories of people that did meditate.

3.3. Depression Risk. Those who had meditated in the last year were less likely to screen positive for depression compared to those who had not $(P<0.001)$. A significant association was found also with frequency of meditation $(P<$ 0.001) (data not shown).
Logistic regression controlling for gender, age, and level of education showed that compared to those who never meditated, those who meditated once or more per week had half the odds of screening positive for depression (Table 3).

3.4. Disability. There was no association between those who did and did not meditate and PDDS score. However, there was a significant association between frequency of meditation and PDDS score $(P<0.001)$, with those who were more disabled meditating more frequently (data not shown).

Ordinal logistic regression was undertaken to model PDDS, controlling for gender, age, and level of education; the model predicted that the odds of increased disability for those who meditated less than once per week were $25 \%$ lower compared to those who never meditated (Table 3). Meditating once or more per week compared to not meditating did not predict disability.

3.5. Clinically Significant Fatigue. There was no significant relationship between those who did and did not meditate and FSS scores. Therefore regression analysis was not explored.

3.6. Relapse Rate. For the subset of people with relapsingremitting MS, there was no effect of ever meditating or frequency of meditation on the mean number of 12-month relapses. Therefore, regression analysis was not used to further explore relapse rate and meditation. 


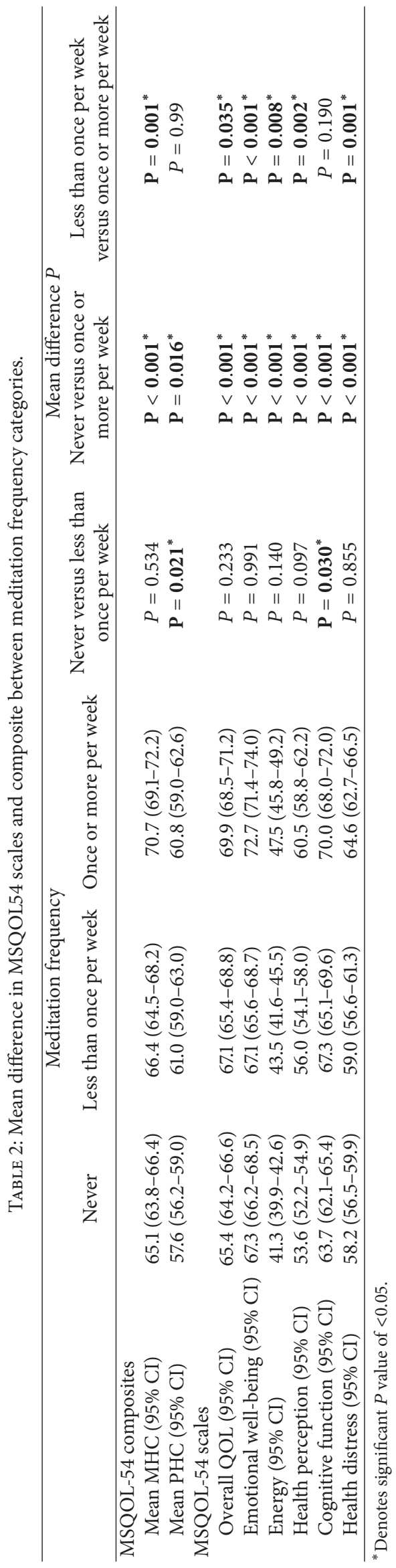


TABLE 3: Predicting the PHC, MHC, and depression with meditation subgroups.

\begin{tabular}{|c|c|c|c|c|c|c|}
\hline \multirow{2}{*}{ Dependent } & \multirow{2}{*}{ Covariates } & \multirow{2}{*}{$B$} & \multicolumn{2}{|c|}{$95 \% \mathrm{CI}$} & \multirow{2}{*}{$P$} & \multirow{2}{*}{ Adjusted $R^{2}$} \\
\hline & & & Lower & Upper & & \\
\hline \multirow{7}{*}{ MHC } & Age, years & 0.10 & 0.02 & 0.19 & 0.022 & \multirow{7}{*}{0.030} \\
\hline & Gender, male & 0.62 & -1.78 & 3.02 & 0.613 & \\
\hline & Secondary school or less* & -7.31 & -9.94 & -4.67 & $<0.001$ & \\
\hline & Vocational training* & -4.45 & -7.42 & -1.49 & 0.003 & \\
\hline & Bachelor degree* & -1.64 & -4.03 & 0.75 & 0.179 & \\
\hline & Meditates once or more per week ${ }^{\dagger}$ & 4.80 & 2.65 & 6.94 & $<0.001$ & \\
\hline & Meditates less than once per week ${ }^{\dagger}$ & 0.78 & -1.55 & 3.11 & 0.513 & \\
\hline \multirow{8}{*}{ PHC } & Age, years & 0.42 & 0.33 & 0.51 & $<0.001$ & \multirow{7}{*}{0.081} \\
\hline & Gender, male & 2.67 & 0.22 & 5.11 & $<0.001$ & \\
\hline & Secondary school or less* & -8.60 & -11.38 & -5.82 & $<0.001$ & \\
\hline & Vocational training* & -7.31 & -10.37 & -4.24 & $<0.001$ & \\
\hline & Bachelor degree ${ }^{*}$ & -2.16 & -2.71 & 2.28 & 0.87 & \\
\hline & Meditates once or more per week ${ }^{\dagger}$ & 2.99 & 0.75 & 5.24 & 0.009 & \\
\hline & Meditates less than once per week ${ }^{\dagger}$ & 2.69 & 0.24 & 5.15 & 0.031 & \\
\hline & & OR & Lower & Upper & $P$ & \\
\hline \multirow{7}{*}{ Depression } & Age, years & 0.99 & 0.98 & 1.00 & 0.092 & \\
\hline & Gender, male & 1.01 & 0.75 & 1.34 & 0.976 & \\
\hline & Secondary school or less ${ }^{\#}$ & 2.07 & 1.49 & 2.88 & $<0.001$ & \\
\hline & Vocational training $^{\#}$ & 1.88 & 1.31 & 2.70 & $<0.001$ & \\
\hline & Bachelor degree $^{\#}$ & 1.27 & 0.92 & 1.75 & 0.146 & \\
\hline & Once or more per week ${ }^{\ddagger}$ & 0.52 & 0.39 & 0.69 & $<0.001$ & \\
\hline & Less than once per week ${ }^{\ddagger}$ & 0.88 & 0.67 & 1.16 & 0.366 & \\
\hline \multirow{7}{*}{ Disability } & Age, years & 1.08 & 1.07 & 1.09 & $<0.001$ & \\
\hline & Gender, male & 1.04 & 0.83 & 1.31 & 0.726 & \\
\hline & Secondary school or less ${ }^{\#}$ & 1.57 & 1.22 & 2.02 & $<0.001$ & \\
\hline & Vocational training $^{\#}$ & 1.72 & 1.30 & 2.27 & $<0.001$ & \\
\hline & Bachelor degree $\mathrm{A}^{\#}$ & 1.04 & 0.82 & 1.31 & 0.773 & \\
\hline & Once or more per week ${ }^{\ddagger}$ & 1.18 & 0.96 & 1.44 & 0.108 & \\
\hline & Less than once per week ${ }^{\ddagger}$ & 0.75 & 0.59 & 0.94 & 0.014 & \\
\hline
\end{tabular}

$B$ is the unstandardized regression coefficient and $95 \%$ CI is the $95 \%$ confidence interval of the unstandardized regression coefficient.

* Compared to postgraduate qualification.

†Compared to "never meditates."

OR is the odds ratio and $95 \% \mathrm{CI}$ is the $95 \%$ confidence interval of the odds ratio.

${ }^{\#}$ Compared to postgraduate qualification.

"Compared to "never meditates."

3.7. Disease Activity. Among people with relapsing-remitting MS, there was no significant association between whether disease activity was decreasing (improving), stable or increasing (worsening), and meditation status or frequency of meditation. Given the bivariate results were insignificant no regression analysis was performed.

\section{Discussion}

This study is the first cross-sectional survey to focus solely on the association between meditation and MS health related outcomes. Well-educated women aged 50-59 with relapsingremitting MS were the most commonly represented demographic in this survey. This is consistent with relapsingremitting MS being the most common form of the disease
[3] and MS being a disease which more commonly affects females (although our sample had an even higher proportion of females than usually seen in MS cohorts) with a typical onset between 20 and 40 years of age [44]. Participants in the study were mostly English speaking from Western countries; however, there was a more culturally and geographically diverse population than in previous similar studies $[25,27]$.

In our study, those most likely to meditate were women, those over 50 years of age, participants who worked part time, and those with bachelor degrees or higher. Just over $50 \%$ of participants reported having meditated at least once in the last 12 months and 30\% reported meditating at least weekly. The rate of meditation in this study is higher than that found in previous papers reporting meditation rates in MS to range from 9 to $25 \%[25-28,45]$. This may be due to the HOLISM 
study recruiting participants from online platforms, including sites which promoted a holistic management approach to MS, resulting in a strong representation of people who were interested in self-management of their condition.

Our study provides support for work done by Grossman et al. [20]; using different methodology in a much larger, geographically diverse sample, we have shown similar associations between meditation and a variety of quality of life outcomes. Meditation was significantly associated with higher mean scores in both the mental and physical health composite as well as in many of the 12 MSQOL-54 scales. HRQOL measurements are derived from the SF-36 and it is generally accepted that an improvement in this scale of five points is clinically significant [46, 47]. As such, a clinically significant benefit in MHC may exist for those who meditated once a week or more compared to those who never meditated. This association held in multivariate analysis controlling for age, gender, and education level.

Meditation was statistically and possibly clinically significantly related to higher health perception and cognitive function subscores. Much of meditation is involved with observing thoughts and emotions and exploring them without becoming reactive to them $[12,16,17]$. These results suggest that being able to explore emotions may assist people with MS to have a better understanding and acceptance of the disease and therefore improved perception of health status. Furthermore, a more relaxed state of mind and increased levels of concentration associated with meditation [19] may allow for improved cognitive function in those with MS who meditate.

Physical health composite scores were found to be significantly higher in meditators on bivariate and multivariate analysis; however the improvement did not satisfy the clinical significance criteria above. As such, more thorough analysis of the relationship between the PHC and meditation status is warranted in future studies.

Similarly, a statistically significant increase was seen in almost all the other MSQOL-54 scales; however other than those above they may not have been clinically significant. The varying efficacy of meditation on different scales and composites of the MSQOL-54 suggests that the relationship between HRQOL and meditation status is a complex one.

Importantly, participants who meditated had markedly lower depression risk. Multivariate analysis supported a significant relationship between frequency of meditation and decreased risk of depression for those who meditated once or more per week compared to those who never meditated. While the results were suggestive, more in depth analysis into the finding may be necessary given that the significance of the effect was lost when participants who meditated less than once a week were compared to participants who never meditated.

Interestingly, those who meditated once or more per week were significantly more likely to have a higher level of disability, as assessed using the PDDS. This finding may suggest that those with greater disability are more likely to meditate, perhaps feeling a greater need to meditate as a result of their condition. Our analyses showed that those aged over 50 years were more likely to meditate, so it may be that many people with MS begin to consider meditation as an adjunct to treatment as they reach middle age and this may coincide with the natural timing of disease progression in MS, a possibility given that age was shown to be a significant variable on regression analysis.

Results for fatigue, relapse rate, and disease activity were less robust. Results for each of these categories were statistically insignificant, with no association demonstrated between relapse rates and disease activity with frequency of meditation. Given that meditation appears to be associated with depression, it is surprising that a similar relationship was not observed with fatigue, as the literature indicates that these two conditions are commonly intertwined [48, 49].

A deeper appreciation of the biological association between meditation and MS disease course is needed, given the mixed results demonstrated in our analysis. A better understanding of the biological relationship will enhance our understanding of meditation as a preventive strategy for those with MS. Cortisol, a hormone secreted by the anterior pituitary in response to stress, may be involved in the mechanism by which practising meditation is able to alter MS disease course. Previous studies have shown a decrease in cortisol after sustained meditation $[50,51]$. Such a decrease in cortisol, which is often elevated in people with chronic conditions such as MS [52], may contribute to improved HRQOL for those who practise meditation. Meditation has also been shown to alter brain structure and function [10-18]. While the mechanisms for these changes remain unknown, this too could be behind the improvement in HRQOL seen in those with MS who practise meditation. Although the exact mechanism remains unknown, this study showed that meditation may be associated with a clinically significant improvement in HRQOL and depression for those with MS. Given these positive findings, further investigation into the relationship between meditation and MS is warranted.

4.1. Limitations. Our data may have been susceptible to recall difficulties given that responses were self-reported and participants were asked questions regarding their disease and habits over long time periods. This would be especially true of recalling number of relapses in the last 12 months and five years as well as frequency of meditation in the last 12 months. Furthermore participants were required to selfreport physician diagnosis of MS, a factor which could not be verified clinically, given the size and geographic diversity of the cohort.

There may have been responder bias given that there were no compulsory questions in the HOLISM survey. As a result respondents who had experience with meditation may have been more likely to answer the questions relating to meditation, although the item response rate is high at over $80 \%$. Furthermore, although data were deidentified after collection, participation was not anonymous which could also have affected participants' tendency to over- or understate their responses based on what they believed the researchers were wanting to achieve. There may have been further response bias as a result of the survey being internet 
based, meaning that respondents were required to have access to the internet and be somewhat computer literate.

Meditation encompasses a wide range of nonhomogenous activities. It is possible that some forms of meditation are more or less effective than others. Given this study did not discriminate between types of meditation, that is, no definition of meditation was given to participants who were simply asked whether or not they meditated, it is possible the results are not generalisable to all forms of meditation.

Reverse causation cannot be excluded. That is, those with an increased HRQOL choose to meditate rather than meditation being a contributing factor in their improved HRQOL. Similarly it could be, although it seems implausible, that meditation leads to greater disability rather than those with a greater disability being more likely to engage in meditation.

\section{Conclusion}

This study reveals a significant association between meditation and better HRQOL and lower risk of depression for those with MS. This further supports the role of lifestyle modification as secondary or tertiary preventive management in MS and highlights the need for further research into the association between meditation and health outcomes in MS. Finally, given our findings and those of others, clinicians may consider encouraging patients with MS to consider meditation as a management strategy.

\section{Conflict of Interests}

George A. Jelinek receives royalties from his book "Overcoming Multiple Sclerosis: An Evidence-Based Guide to Recovery."

\section{Acknowledgments}

The authors thank all the participants of the HOLISM study and the Bloom Foundation and the Horne Foundation for generous support of the project: this project was funded by the Bloom Foundation and the Horne Family Foundation.

\section{References}

[1] D. H. Miller and S. M. Leary, "Primary-progressive multiple sclerosis," The Lancet Neurology, vol. 6, no. 10, pp. 903-912, 2007.

[2] C. Confavreux, S. Vukusic, T. Moreau, and P. Adeleine, "Relapses and progression of disability in multiple sclerosis," The New England Journal of Medicine, vol. 343, no. 20, pp. 14301438, 2000.

[3] F. D. Lublin and S. C. Reingold, "Defining the clinical course of multiple sclerosis: results of an international survey," Neurology, vol. 46, no. 4, pp. 907-911, 1996.

[4] A. B. Ben-Zacharia, "Therapeutics for multiple sclerosis symptoms," Mount Sinai Journal of Medicine, vol. 78, no. 2, pp. 176191, 2011.

[5] I. S. Haussleiter, M. Brune, and G. Juckel, "Psychopathology in multiple sclerosis: diagnosis, prevalence and treatment,"
Therapeutic Advances in Neurological Disorders, vol. 2, no. 1, pp. 13-29, 2009.

[6] A. J. Thompson, A. T. Toosy, and O. Ciccarelli, "Pharmacological management of symptoms in multiple sclerosis: current approaches and future directions," The Lancet Neurology, vol. 9, no. 12, pp. 1182-1199, 2010.

[7] D. C. Mohr, D. E. Goodkin, P. Bacchetti et al., "Psychological stress and the subsequent appearance of new brain MRI lesions in MS," Neurology, vol. 55, no. 1, pp. 55-61, 2000.

[8] K. D. Ackerman, R. Heyman, B. S. Rabin et al., "Stressful life events precede exacerbations of multiple sclerosis," Psychosomatic Medicine, vol. 64, no. 6, pp. 916-920, 2002.

[9] J. Kabat-Zinn, "An outpatient program in behavioral medicine for chronic pain patients based on the practice of mindfulness meditation: theoretical considerations and preliminary results," General Hospital Psychiatry, vol. 4, no. 1, pp. 33-47, 1982.

[10] A. Chiesa and A. Serretti, "A systematic review of neurobiological and clinical features of mindfulness meditations," Psychological Medicine, vol. 40, no. 8, pp. 1239-1252, 2010.

[11] H. Wahbeh, S.-M. Elsas, and B. S. Oken, "Mind-body interventions: applications in neurology," Neurology, vol. 70, no. 24, pp. 2321-2328, 2008.

[12] P. Grossman, L. Niemann, S. Schmidt, and H. Walach, "Mindfulness-based stress reduction and health benefits: a meta-analysis," Journal of Psychosomatic Research, vol. 57, no. 1, pp. 35-43, 2004.

[13] R. A. Baer, "Mindfulness training as a clinical intervention: a conceptual and empirical review," Clinical Psychology: Science and Practice, vol. 10, no. 2, pp. 125-143, 2003.

[14] A. Chiesa and A. Serretti, "Mindfulness based cognitive therapy for psychiatric disorders: a systematic review and metaanalysis," Psychiatry Research, vol. 187, no. 3, pp. 441-453, 2011.

[15] T. S. Mars and H. Abbey, "Mindfulness meditation practise as a healthcare intervention: a systematic review," International Journal of Osteopathic Medicine, vol. 13, no. 2, pp. 56-66, 2010.

[16] S.-L. Keng, M. J. Smoski, and C. J. Robins, "Effects of mindfulness on psychological health: a review of empirical studies," Clinical Psychology Review, vol. 31, no. 6, pp. 1041-1056, 2011.

[17] P. Vestergaard-Poulsen, M. Van Beek, J. Skewes et al., "Longterm meditation is associated with increased gray matter density in the brain stem," NeuroReport, vol. 20, no. 2, pp. 170-174, 2009.

[18] Y.-Y. Tang, Q. Lu, M. Fan, Y. Yang, and M. I. Posner, "Mechanisms of white matter changes induced by meditation," Proceedings of the National Academy of Sciences of the United States of America, vol. 109, no. 26, pp. 10570-10574, 2012.

[19] A. Senders, D. Bourdette, D. Hanes, V. Yadav, and L. Shinto, "Perceived stress in multiple sclerosis: the potential role of mindfulness in health and well-being," Journal of EvidenceBased Complementary and Alternative Medicine, vol. 19, no. 2, pp. 104-111, 2014.

[20] P. Grossman, L. Kappos, H. Gensicke et al., "MS quality of life, depression, and fatigue improve after mindfulness training: a randomized trial," Neurology, vol. 75, no. 13, pp. 1141-1149, 2010.

[21] J. Tavee, M. Rensel, S. Pope Planchon, and L. Stone, "Effects of meditation on pain and quality of life in multiple sclerosis and polyneuropathy: a controlled study," International Journal of MS Care, vol. 13, supplement 2, pp. 163-168, 2011.

[22] N. Mills and J. Allen, "Mindfulness of movement as a coping strategy in multiple sclerosis. A pilot study," General Hospital Psychiatry, vol. 22, no. 6, pp. 425-431, 2000. 
[23] M. P.-M. Li, G. A. Jelinek, T. J. Weiland et al., "Effect of a residential retreat promoting lifestyle modifications on healthrelated quality of life in people with multiple sclerosis," Quality in Primary Care, vol. 18, no. 6, pp. 379-389, 2010.

[24] E. J. Hadgkiss, G. A. Jelinek, T. J. Weiland et al., "Health-related quality of life outcomes at 1 and 5 years after a residential retreat promoting lifestyle modification for people with multiple sclerosis," Neurological Sciences, vol. 34, no. 2, pp. 187-195, 2013.

[25] L. Skovgaard, P. H. Nicolajsen, E. Pedersen et al., "Differences between users and non-users of complementary and alternative medicine among people with multiple sclerosis in Denmark: a comparison of descriptive characteristics," Scandinavian Journal of Public Health, vol. 41, no. 5, pp. 492-499, 2013.

[26] R. D. Simmons, A.-L. Ponsonby, I. A. F. van der Mei, and P. Sheridan, "What affects your MS? Responses to an anonymous, internet-based epidemiological survey," Multiple Sclerosis, vol. 10, no. 2, pp. 202-211, 2004.

[27] S. Nayak, R. J. Matheis, N. E. Schoenberger, and S. C. Shiflett, "Use of unconventional therapies by individuals with multiple sclerosis," Clinical Rehabilitation, vol. 17, no. 2, pp. 181-191, 2003.

[28] L. Esmonde and A. F. Long, "Complementary therapy use by persons with multiple sclerosis: benefits and research priorities," Complementary Therapies in Clinical Practice, vol. 14, no. 3, pp. 176-184, 2008.

[29] G. A. Jelinek, E. J. Hadgkiss, T. J. Weiland, N. G. Pereira, C. H. Marck, and D. M. Van Der Meer, "Association of fish consumption and omega 3 supplementation with quality of life, disability and disease activity in an international cohort of people with multiple sclerosis," International Journal of Neuroscience, vol. 123, no. 11, pp. 792-801, 2013.

[30] E. J. Hadgkiss, G. A. Jelinek, T. J. Weiland, N. G. Pereira, C. H. Marck, and D. M. van der Meer, "The association of diet with quality of life, disability, and relapse rate in an international sample of people with multiple sclerosis," Nutritional Neuroscience, 2014.

[31] T. J. Weiland, E. J. Hadgkiss, G. A. Jelinek, N. G. Pereira, C. H. Marck, and D. M. Van Der Meer, "The association of alcohol consumption and smoking with quality of life, disability and disease activity in an international sample of people with multiple sclerosis," Journal of the Neurological Sciences, vol. 336, no. 1-2, pp. 211-219, 2014.

[32] E. J. Hadgkiss, G. A. Jelinek, T. J. Weiland, N. G. Pereira, C. H. Marck, and D. M. van der Meer, "Methodology of an international study of people with multiple sclerosis recruited through web 2.0 platforms: demographics, lifestyle, and disease characteristics," Neurology Research International, vol. 2013, Article ID 580596, 12 pages, 2013.

[33] A. Solari, G. Filippini, L. Mendozzi et al., "Validation of Italian multiple sclerosis quality of life 54 questionnaire," Journal of Neurology Neurosurgery and Psychiatry, vol. 67, no. 2, pp. 158$162,1999$.

[34] T. Yamamoto, K. Ogata, M. Katagishi et al., "Validation of the Japanese-translated version multiple sclerosis quality of life-54 instrument," Clinical Neurology, vol. 44, no. 7, pp. 417-421, 2004.

[35] C. Acquardo, L. Lafortune, and I. Mear, "Quality of life in multiple sclerosis: translation in French Canadian of the MSQoL-54," Health and Quality of Life Outcomes, vol. 1, article 70, 2003.

[36] M. J. Hohol, E. J. Orav, and H. L. Weiner, "Disease steps in multiple sclerosis: a simple approach to evaluate disease progression," Neurology, vol. 45, no. 2, pp. 251-255, 1995.

[37] Y. C. Learmonth, R. W. Motl, B. M. Sandroff, J. H. Pula, and D. Cadavid, "Validation of patient determined disease steps
(PDDS) scale scores in persons with multiple sclerosis," $B M C$ Neurology, vol. 13, article 37, 2013.

[38] M. J. Hohol, E. J. Orav, and H. L. Weiner, "Disease steps in multiple sclerosis: a longitudinal study comparing Disease Steps and EDSS to evaluate disease progression," Multiple Sclerosis, vol. 5, no. 5, pp. 349-354, 1999.

[39] R. A. Marrie and M. Goldman, "Validity of performance scales for disability assessment in multiple sclerosis," Multiple Sclerosis, vol. 13, no. 9, pp. 1176-1182, 2007.

[40] K. Kroenke, R. L. Spitzer, and J. B. W. Williams, "The patient health questionnaire-2: validity of a two-item depression screener," Medical Care, vol. 41, no. 11, pp. 1284-1292, 2003.

[41] P. O. Valko, C. L. Bassetti, K. E. Bloch, U. Held, and C. R. Baumann, "Validation of the fatigue severity scale in a Swiss cohort," Sleep, vol. 31, no. 11, pp. 1601-1607, 2008.

[42] L. B. Krupp, N. G. LaRocca, J. Muir-Nash, and A. D. Steinberg, "The fatigue severity scale. Application to patients with multiple sclerosis and systemic lupus erythematosus," Archives of Neurology, vol. 46, no. 10, pp. 1121-1123, 1989.

[43] H. Kaynak, A. Altintaş, D. Kaynak et al., "Fatigue and sleep disturbance in multiple sclerosis," European Journal of Neurology, vol. 13, no. 12, pp. 1333-1339, 2006.

[44] R. G. Richards, F. C. Sampson, S. M. Beard, and P. Tappenden, "A review of the natural history and epidemiology of multiple sclerosis: implications for resource allocation and health economic models," Health Technology Assessment, vol. 6, no. 10, pp. $1-73,2002$.

[45] C. S. Berkman, M. G. Pignotti, P. F. Cavallo, and N. J. Holland, "Use of alternative treatments by people with multiple sclerosis," Neurorehabilitation and Neural Repair, vol. 13, no. 4, pp. 243254, 1999.

[46] L. Kappos, R. Gold, D. L. Arnold et al., "Quality of life outcomes with BG-12 (dimethyl fumarate) in patients with relapsingremitting multiple sclerosis: the DEFINE study," Multiple Sclerosis, vol. 20, no. 2, pp. 243-252, 2014.

[47] G. R. Norman, J. A. Sloan, and K. W. Wyrwich, "Interpretation of changes in health-related quality of life the remarkable universality of half a standard deviation," Medical Care, vol. 41, no. 5, pp. 582-592, 2003.

[48] R. Bakshi, Z. A. Shaikh, R. S. Miletich et al., "Fatigue in multiple sclerosis and its relationship to depression and neurologic disability," Multiple Sclerosis, vol. 6, no. 3, pp. 181-185, 2000.

[49] B. Wood, I. A. F. van der Mei, A.-L. Ponsonby et al., "Prevalence and concurrence of anxiety, depression and fatigue over time in multiple sclerosis," Multiple Sclerosis, vol. 19, no. 2, pp. 217-224, 2013.

[50] S. Brand, E. Holsboer-Trachsler, J. R. Naranjo, and S. Schmidt, "Influence of mindfulness practice on cortisol and sleep in longterm and short-term meditators," Neuropsychobiology, vol. 65, no. 3, pp. 109-118, 2012.

[51] R. H. Matousek, P. L. Dobkin, and J. Pruessner, "Cortisol as a marker for improvement in mindfulness-based stress reduction," Complementary Therapies in Clinical Practice, vol. 16, no. 1, pp. 13-19, 2010.

[52] D. C. Mohr, "Stress and multiple sclerosis," Journal of Neurology, vol. 254, supplement 2, pp. II65-II68, 2007. 


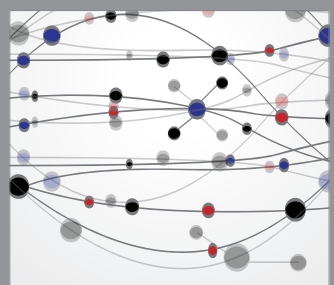

The Scientific World Journal
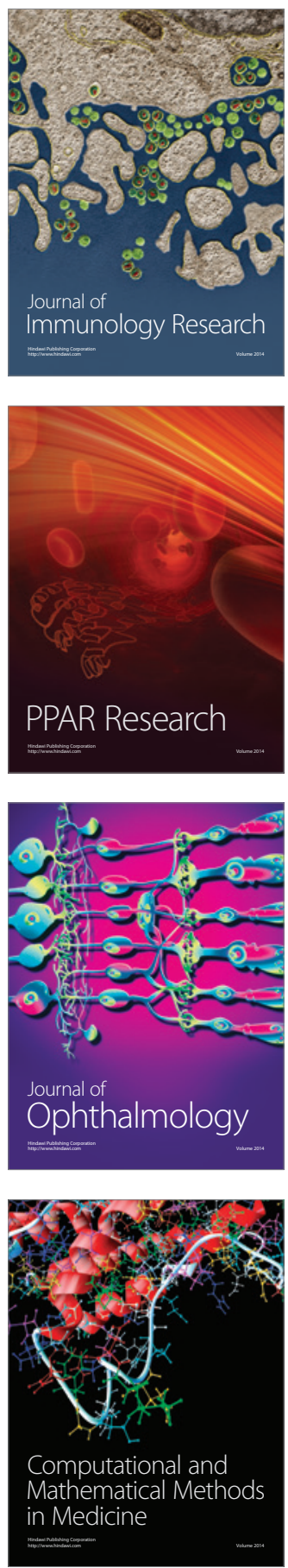

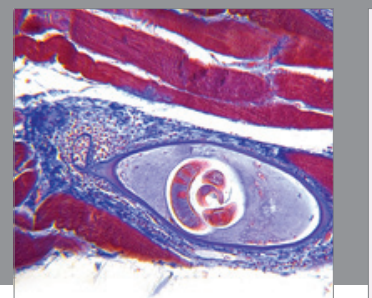

Gastroenterology

Research and Practice
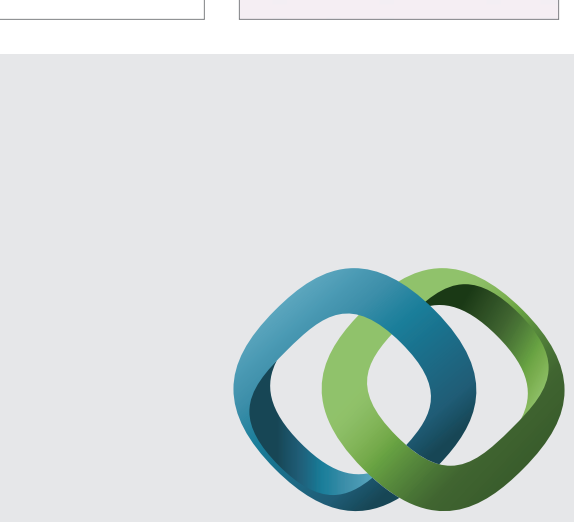

\section{Hindawi}

Submit your manuscripts at

http://www.hindawi.com
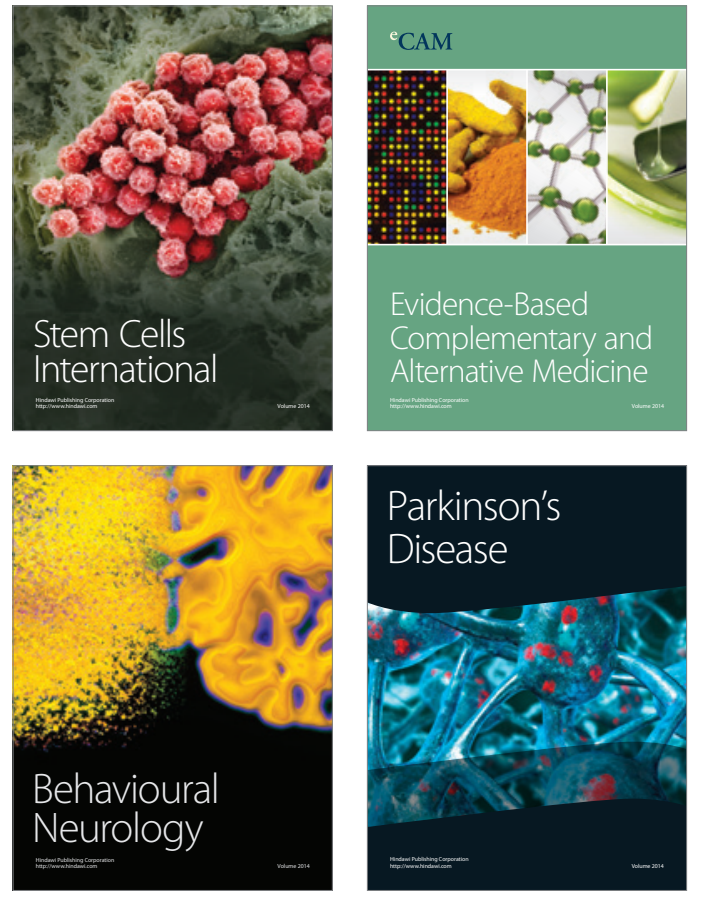
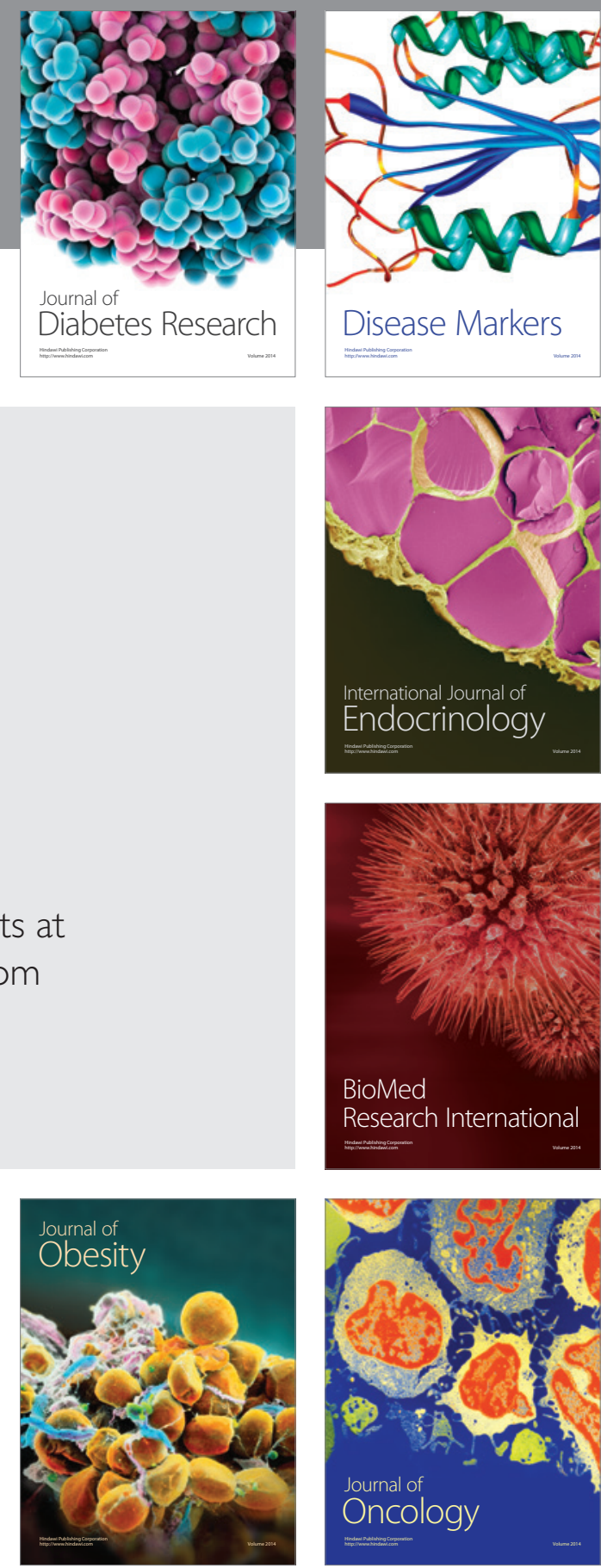

Disease Markers
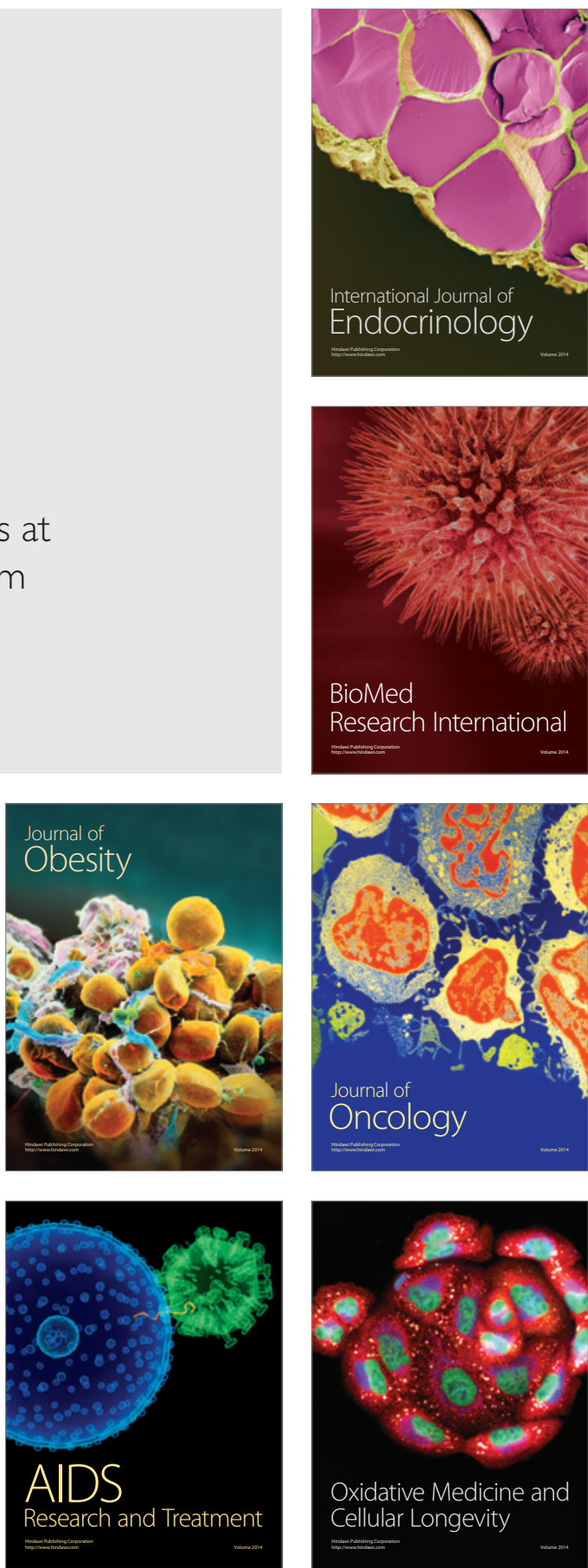